\title{
La gestión ágil de proyectos de software en la formación Académica Universitaria: Una revisión sistemática de la literatura
}

\section{Agile management of software projects in university academic training: A systemic review of literature}

\author{
HERNÁNDEZ-CRUZ，Luz María†*，CASTILLO-TÉLLEZ，Margarita，MEX-ALVAREZ，Diana \\ Concepción y CAB-CHAN José Ramón
}

Universidad Autónoma de Campeche. Facultad de Ingeniería.

ID $1^{\text {er }}$ Autor: Luz María, Hernández-Cruz / ORC ID: https://orcid.org/0000-0002-0469-5298, Researcher ID Thomson: H3153-2018, CVU CONACYT ID: 662220

ID $1^{\mathrm{er}}$ Coautor: Margarita, Castillo-Téllez, ORC ID: 0000-0001-9639-1736, Researcher ID Thomson: S-2283-2018, CVU CONACYT ID: 210428

ID $2^{\text {do }}$ Coautor: Diana Concepción, Mex-Álvarez / ORC ID: 0000-0001-9419-7868, Researcher ID Thomson: I-4164-2018, CVU CONACYT ID: 842039

ID $3^{\text {er }}$ Coautor: José Ramón Cab-Chan / ORC ID: 0000-0003-1043-629, Researcher ID Thomson: I-5425-2018, CVU CONACYT ID: 204250

\section{Resumen}

La gestión ágil de proyectos es un enfoque iterativo que permiten planificar y guiar los procesos de proyectos que precisan de una especial rapidez y flexibilidad para alcanzar el éxito de los mismos. Es por ello, que hoy día los profesionistas deben dominar como competencia disciplinar o profesionalizante los conceptos base de esta labor. El objetivo del estudio radica principalmente en recopilar investigaciones recientes, incluyendo los últimos tres años, con relación a la Gestión Ágil de Proyectos de Software, su situación actual y su aplicación en la práctica. En el estudio se ostenta la implementación de la metodología propuesta por Kitchenham para la revisión sistemática de la literatura (RSL) analizando 40 publicaciones científicas, concluyendo que la gestión ágil de proyectos de software permite la gestión eficiente y eficaz con la aplicación de un estándar o metodología prioritaria y con el uso ineludible de herramientas de software de carácter específico para ello.

Revisión sistemática de literatura, Gestión ágil de proyectos, Software

\begin{abstract}
Agile project management is an iterative approach that allows you to plan and guide project processes that require special speed and flexibility to achieve project success. It is for this reason that today professionals must master the basic concepts of this work as disciplinary or professionalizing competence. The objective of the study lies mainly in compiling recent research, including the last three years, in relation to Agile Software Project Management, its current situation and its application in practice. The study shows the implementation of the methodology proposed by Kitchenham for the systematic literature review (RSL) analyzing 40 scientific publications, concluding that the agile management of software projects allows efficient and effective management with the application of a standard o priority methodology and with the unavoidable use of software tools of specific use for it.
\end{abstract}

Systematic literature review, Agile project management, Software

Citación: HERNÁNDEZ-CRUZ, Luz María, CASTILLO-TÉLLEZ, Margarita, MEX-ALVAREZ, Diana Concepción y CAB-CHAN José Ramón. La gestión ágil de proyectos de software en la formación Académica Universitaria: Una revisión sistemática de la literatura. Revista de Tecnología y Educación. 2020. 4-11: 8-21

\footnotetext{
$\dagger$ Investigador contribuyendo como primer autor.
} 


\section{Introducción}

La gestión ágil de proyectos ha sido desarrollada como un nuevo paradigma para la administración de proyectos dinámicos, permitiendo a empresas y clientes crear valor a través de metodologías ágiles durante el ciclo de vida de productos de software. Este enfoque ha sido ampliamente aceptado en el ámbito empresarial, no siendo aplicado en profundidad en el contexto de la formación universitaria. Por esta razón, el principal aporte del presente estudio es profundizar, mediante una revisión sistemática de la literatura, en el concepto de la gestión ágil de proyectos de software y su aplicación en la práctica, contribuyendo a una visión clara del quehacer disciplinar. De igual forma, se busca la relación del estándar internacional PMBOK como guía para la gestión ágil de proyectos.

El estudio genera valor agregado con el uso de la revisión sistemática de la literatura como una nueva opción en la recopilación y análisis de bibliografía en el área de ingeniería de software, destacando además de envolver fuentes científicas de impacto en el área a tratar. Las secciones principales del artículo son:

Fase I: Planificar la revisión sistemática de la literatura. Comprende la especificación de las preguntas de investigación, el desarrollo y validación del protocolo de la revisión.

Fase II: Conducir la revisión sistemática de la literatura. Comprende la identificación de las fuentes científicas, seleccionar los estudios relevantes, evaluar la calidad de los estudios seleccionados, extraer y sintetizar los datos.

Fase III: Documentar la revisión sistemática de la literatura. Escribir y validar el informe de la revisión.

\section{Metodología}

La Revisión Sistemática de la Literatura (RSL) se fundamenta en la metodología propuesta por Bárbara Kitchenham. El proceso global de la revisión consiste en tres fases: Planificar la RSL, Conducir la RSL y documentar la RSL. La Figura 1 muestra el proceso de estudio para una Revisión Sistemática de la Literatura (RSL).

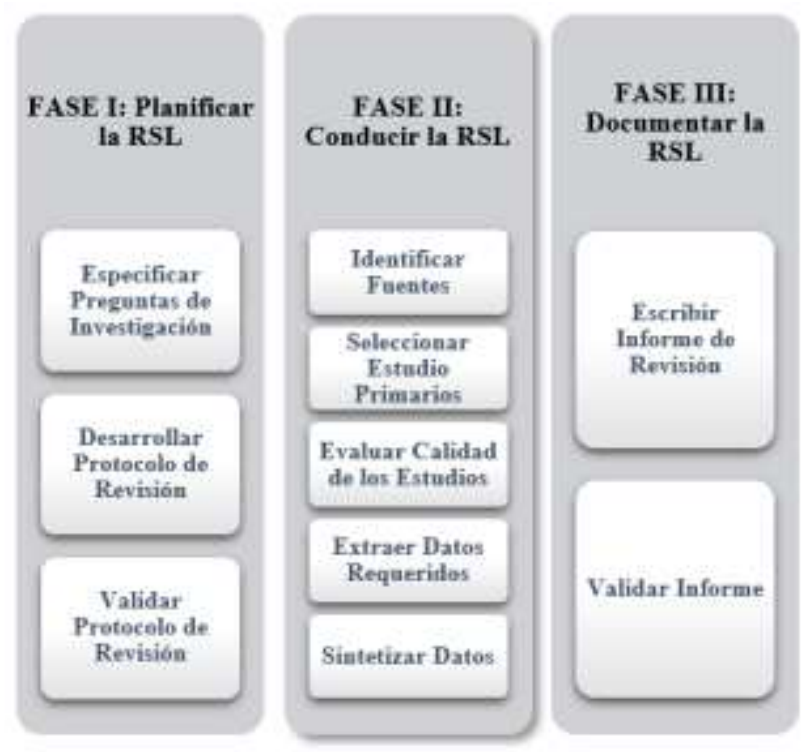

Figura 1 Estudio de Revisión Sistemática de la Literatura Fuente: traducido de (Kitchenham, Budgen, \& Brereton, 2015)

\section{Fase I: Planificar de la RSL}

\section{Especificar las Preguntas de Investigación}

Las preguntas de investigación son las metas que se buscan responder y son la guía durante todo el proceso de investigación. Las preguntas de investigación propuestas son:

- $\quad$ Pregunta 1 (P1): ¿Cuál es el papel de la Gestión de Proyectos en la actualidad?

- Pregunta 2 (P2): ¿Cuáles son los atributos relacionados con la Gestión Ágil de Proyectos?

- $\quad$ Pregunta 3 (P3): ¿Cómo se adopta el estándar PMBOK en la Gestión Ágil de Proyectos?

- $\quad$ Pregunta 4 (P4): ¿Cómo llevar a cabo una Gestión Ágil de Proyectos en el Desarrollo de Software como parte de la Formación Académica Universitaria?

El objetivo general de la investigación consiste en realizar una revisión sistemática de la literatura que permita encontrar estudios relacionados con "La Gestión Ágil de Proyectos de Software”. La búsqueda se delimita en un espacio temporal del 01 de enero de 2017 al 31 de marzo de 2020, esto considerando como origen de partida la necesidad de la RSL para resumir la investigación realizada en campos de la ingeniería de software, que tienen un desarrollo rápido y se están expandiendo constantemente, tal que, además las revisiones previas se hacen obsoletas rápidamente. 


\section{Desarrollar el Protocolo de Revisión Términos de búsqueda}

Los términos de búsqueda son específicamente palabras o frases cortas que permitan encontrar artículos enfocados a resolver las preguntas de investigación planteadas. La Tabla 1 muestra los principales términos de la investigación y sus textos alternativos.

\begin{tabular}{|l|l|}
\hline \multicolumn{2}{|c|}{ Término Textos alternativos } \\
\hline Proyecto & No aplica \\
\hline Gestión & Dirección, Administración \\
\hline Éxito & No aplica \\
\hline Ágil & No aplica \\
\hline PMBOK & No aplica \\
\hline Software & $\begin{array}{l}\text { Sistemas de Información, Sistemas } \\
\text { informáticos }\end{array}$ \\
\hline
\end{tabular}

Tabla 1 Términos de Búsqueda en la presente RSL Fuente: Elaboración Propia

A partir de los términos de búsqueda se obtienen las cadenas de búsquedas. La Tabla 2 muestra las cadenas de búsquedas para la presente RSL.

\begin{tabular}{|l|l|}
\hline \multicolumn{1}{|c|}{ Término } & \multicolumn{1}{c|}{ Cadenas De Búsqueda } \\
\hline Simples & Proyecto/Project \\
\hline Proyecto & $\begin{array}{l}\text { Gestión/Administración/Dirección } \\
\text { Administración de proyectos/Dirección } \\
\text { de proyectos/Project Management }\end{array}$ \\
\hline Gestión & Éxito de proyectos/ Project success \\
\hline Éxito & $\begin{array}{l}\text { Ágil/Agile } \\
\text { Agilidad }\end{array}$ \\
\hline Ágil & $\begin{array}{l}\text { PMBOK } \\
\text { PMBÓndar v6.0 } \\
\text { PMBOK }\end{array}$ \\
& $\begin{array}{l}\text { Guía de los fundamentos para la } \\
\text { dirección de } \\
\text { Management Body of Knowledge }\end{array}$ \\
\hline Software & $\begin{array}{l}\text { Software, Sistema de información, } \\
\text { Information System }\end{array}$ \\
\hline Compuestas & $\begin{array}{l}\text { Gestión AND Proyecto } \\
\text { Management AND Project } \\
\text { Ágil AND Proyecto AND Gestión } \\
\text { Agile AND Project AND Management } \\
\text { Ágil AND Proyecto AND Gestión } \\
\text { AND Software } \\
\text { Agile AND Project AND Management } \\
\text { AND Software } \\
\text { Ágil AND Proyecto AND Gestión } \\
\text { AND Software AND PMBOK } \\
\text { Agile AND Project AND Management } \\
\text { AND Software AND PMBOK }\end{array}$ \\
\hline
\end{tabular}

Tabla 2 Cadenas de Búsqueda en la presente RSL Fuente: Elaboración Propia

\section{Estrategia de Búsqueda}

Una estrategia de búsqueda define el acceso al campo de registro por el cual es posible recuperar información. Las fuentes científicas asignadas para la investigación son Bibliotecas Digitales, con prestigio internacional en el área de estudio, y Bases de datos indexadas reconocidas. En este punto, los investigadores apoyados por el juicio de expertos de la Facultad de Ingeniería de la Universidad Autónoma de Campeche definen las Fuentes científicas a considerar. La Tabla 3 muestra las bibliotecas digitales y bases de datos indexadas como fuentes científicas para la presente RSL.

\begin{tabular}{|c|c|}
\hline Fuente & Nombre del artículo \\
\hline \multirow{2}{*}{ Bibliotecas digitales } & IEEE Xplore Digital Library \\
\hline & ACM Digital Library \\
\hline \multirow[t]{2}{*}{ Base de datos indexadas } & DOAJ \\
\hline & SpringerLink \\
\hline
\end{tabular}

Tabla 3 Estrategia de Búsqueda en la presente RSL Fuente: Elaboración Propia

\section{Validar el Protocolo de Revisión}

\section{Criterios de inclusión y exclusion}

La definición de criterios de inclusión y exclusión establecen la pertinencia para la selección de la documentación que formará parte del estudio de la presente investigación. Con lo anterior, se realiza la segmentación y se reduce el volumen de la información.

Los criterios de inclusión son:

- Artículos publicados en revistas científicas.

- $\quad$ Artículos científicos publicados entre los años 2017 y el primer trimestre del año 2020.

- $\quad$ Artículos escritos en inglés y español.

Artículos que respondan completa o parcialmente a una o más de las preguntas de la investigación definidas en la Fase I de la presente RSL.

Los criterios de la exclusión son:

- $\quad$ Artículos que no estén relacionados con el tema "La gestión ágil de proyectos de software". 
- Artículos que no respondan a ninguna pregunta de investigación de la presente RSL.

- $\quad$ Artículos que no se encuentren en las Bibliotecas digitales y Bases de datos indexadas establecidas en el apartado Estrategia de Búsqueda de la presente RSL.

- $\quad$ Artículos duplicados.

\section{Fase II: Conducir la RSL}

\section{Identificar Fuentes o Estudios Relevantes y Selección de Estudios Primarios}

Para iniciar el estudio se realizan las búsquedas aplicando las especificaciones de la Fase I. La Tabla 4 muestra la recopilación de los artículos científicos resultado de las cadenas de búsquedas (Tabla 2) en las Bibliotecas digitales y Bases de datos indexadas establecidas (Tabla 3) de la presente RSL, como primera instancia, sin aplicar los criterios de inclusión y exclusión.

\begin{tabular}{|c|c|}
\hline Fuente & $\mathrm{N}^{\circ}$ de artículos \\
\hline \multicolumn{2}{|l|}{ Bibliotecas digitales } \\
\hline IEEE Xplore Digital Library & 25,866 \\
\hline ACM Digital Library & 362 \\
\hline \multicolumn{2}{|l|}{ Base de datos indexadas } \\
\hline DOAJ & 106 \\
\hline SpringerLink & 157 \\
\hline Total de artículos & 26,491 \\
\hline
\end{tabular}

Tabla 4 Resultado de la primera búsqueda de la presente RSL

Fuente: Elaboración Propia

Los resultados encontrados (artículos que cumplen con las cadenas de búsqueda) se analizan validando su pertinencia con los criterios de inclusión y exclusión definidos para obtener el subconjunto de artículos científicos a incluir en el estudio. La Tabla 5 muestra el subconjunto de artículos que corresponden a los criterios de inclusión y exclusión definidos.

\begin{tabular}{|c|c|}
\hline Fuente & $\mathbf{N}^{\circ}$ de artículos \\
\hline \multicolumn{2}{|l|}{ Bibliotecas digitales } \\
\hline IEEE Xplore Digital Library & 20 \\
\hline ACM Digital Library & 8 \\
\hline \multicolumn{2}{|l|}{ Base de datos indexadas } \\
\hline DOAJ & 7 \\
\hline SpringerLink & 5 \\
\hline Total de artículos & 40 \\
\hline
\end{tabular}

Tabla 5 Subconjunto de artículos científicos para el estudio de la RSL

Fuente: Fuente Propia

El $70 \%$ de artículos científicos del subconjunto de estudio provienen de Bibliotecas digitales, específicamente $50 \%$ de la biblioteca IEEE Xplore Digital Library y 20\% de ACM Digital Library. El 30\% restante de Bases de datos indexadas, con el $17.5 \%$ correspondiente a DOAJ (Directory Of Access Journals) y el $12.5 \%$ restante a SpringerLink. La Gráfica 1 muestra el porcentaje de artículos organizados por fuente.

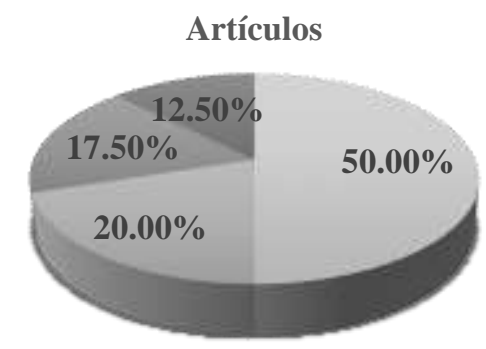

$\square$ IEEE Xplore Digital Library $\square$ ACM Digital Library

- DOAJ $\quad$ SpringerLink

Gráfico 1 Artículos científicos seleccionados para el estudio de la RSL

Fuente: Elaboración Propia

\section{Evaluar la Calidad de los Estudios}

Enseguida, se evalúa la calidad de los artículos encontrados. Los artículos científicos encontrados en Bibliotecas Digitales se clasifican según la revista donde fue publicado y el año de publicación, añadiendo el factor de impacto de la revista y la cantidad de artículos incluidos en el estudio. La Tabla 6 muestra la clasificación de artículos de las Bibliotecas Digitales IEEE Xplore y ACM recopilados para el estudio de la presente RSL. 


\begin{tabular}{|c|c|c|c|}
\hline Revista & Año & $\begin{array}{c}\text { Factor de } \\
\text { impacto/ } \\
\text { Cuartil }\end{array}$ & $\begin{array}{c}\mathbf{N}^{\circ} \text { de } \\
\text { artículos }\end{array}$ \\
\hline \multicolumn{4}{|c|}{ IEEE Xplore Digital Library } \\
\hline IEEE Access & 2019 & $\begin{array}{l}4.098 \\
\text { Q1 }\end{array}$ & 1 \\
\hline IEEE Access & 2020 & $\begin{array}{l}4.098 \\
\text { Q1 }\end{array}$ & 1 \\
\hline $\begin{array}{ll}\text { IEEE } & \text { Engineering } \\
\text { Management Review }\end{array}$ & 2017 & $\begin{array}{l}0.390 \\
\mathrm{Q} 4\end{array}$ & 2 \\
\hline $\begin{array}{ll}\text { IEEE } & \text { Engineering } \\
\text { Management Review }\end{array}$ & 2018 & $\begin{array}{l}0.390 \\
\mathrm{Q} 4\end{array}$ & 3 \\
\hline $\begin{array}{ll}\text { IEEE } & \text { Engineering } \\
\text { Management Review }\end{array}$ & 2019 & $\begin{array}{l}0.390 \\
\mathrm{Q} 4\end{array}$ & 2 \\
\hline IEEE Software & 2017 & $\begin{array}{l}2.945 \\
\mathrm{Q} 2 \\
\end{array}$ & 1 \\
\hline IEEE Software & 2018 & $\begin{array}{l}2.945 \\
\text { Q2 }\end{array}$ & 1 \\
\hline IEEE Software & 2019 & $\begin{array}{l}2.945 \\
\text { Q2 }\end{array}$ & 5 \\
\hline $\begin{array}{l}\text { IEEE Transactions on } \\
\text { Education }\end{array}$ & 2018 & $\begin{array}{l}2.214 \\
\text { Q1 }\end{array}$ & 1 \\
\hline $\begin{array}{ll}\text { IEEE Transactions } & \text { on } \\
\text { Professional } & \\
\text { Communication } & \\
\end{array}$ & 2019 & $\begin{array}{l}1.143 \\
\mathrm{Q} 1\end{array}$ & 1 \\
\hline $\begin{array}{lcc}\text { IEEE Transactions } & \text { on } \\
\text { Software Engineering } & \end{array}$ & 2018 & $\begin{array}{l}4.778 \\
\text { Q1 }\end{array}$ & 1 \\
\hline IET Software & 2019 & $\begin{array}{l}0.695 \\
\text { Q3 }\end{array}$ & 1 \\
\hline \multicolumn{4}{|l|}{ ACM Digital Library } \\
\hline $\begin{array}{l}\text { Journal of Computing } \\
\text { Sciences in Colleges }\end{array}$ & 2017 & No aplica & 3 \\
\hline $\begin{array}{l}\text { Journal of Computing } \\
\text { Sciences in Colleges }\end{array}$ & 2018 & No aplica & 3 \\
\hline $\begin{array}{l}\text { Journal of Computing } \\
\text { Sciences in Colleges }\end{array}$ & 2019 & No aplica & 2 \\
\hline Total de artículos & & & 28 \\
\hline
\end{tabular}

Tabla 6 Selección de artículos obtenidos de las Bibliotecas Digitales IEEE Xplore y ACM para el estudio de la presente RSL. Factor de Impacto de las Revistas Fuente: Elaboración Propia

Los artículos recopilados de las bases de datos indexadas tienen como valor agregado la publicación reciente (incluye los tres primeros meses del presente año). La Tabla 7 muestra el resumen de los artículos recopilados de las Bases de datos DOAJ y SpringerLink para el estudio de la presente RSL.

\begin{tabular}{|l|r|r|}
\hline \multicolumn{1}{|c}{ Base de Datos } & \multicolumn{1}{c|}{ Año } & $\begin{array}{c}\text { No de } \\
\text { artículos }\end{array}$ \\
\hline DOAJ & 2019 & 2 \\
\hline DOAJ & 2020 & 5 \\
\hline SpringerLink & 2018 & 2 \\
\hline SpringerLink & 2019 & 2 \\
\hline SpringerLink & 2020 & 1 \\
\hline Total de artículos & & 12 \\
\hline
\end{tabular}

Tabla 7 Resumen de los artículos obtenidos de las Bases de datos DOAJ y SpringerLink para la presente RSL Fuente: Elaboración Propia

Antes de realizar cualquier análisis es crucial poder identificar la correspondencia o relación de cada artículo científico seleccionado con alguna de las preguntas de investigación definidas en la Fase I.
La Tabla 8 muestra el concentrado de la cantidad de artículos seleccionados en correspondencia a las preguntas de investigación definidas para el estudio.

\begin{tabular}{|l|r|r|r|r|r|}
\hline \multicolumn{2}{|c|}{ P1 } & P2 & \multicolumn{1}{c|}{ P3 } & P4 & \multicolumn{1}{c|}{$\begin{array}{c}\mathbf{N}^{\circ} \text { de } \\
\text { artículos }\end{array}$} \\
\hline $\begin{array}{l}\text { IEEE Xplore } \\
\text { Digital Library }\end{array}$ & 8 & 7 & 2 & 3 & 20 \\
\hline $\begin{array}{l}\text { ACM Digital } \\
\text { Library }\end{array}$ & 0 & 1 & 0 & 7 & 8 \\
\hline DOAJ & 1 & 1 & 1 & 4 & 7 \\
\hline SpringerLink & 3 & 1 & 0 & 1 & 5 \\
\hline Total de artículos & 12 & 10 & 3 & 15 & 40 \\
\hline
\end{tabular}

Tabla 8 Correspondencia de los artículos científicos seleccionados para el estudio con las preguntas de investigación definidas en la presente RSL

Fuente: Elaboración Propia

\section{Extraer Datos Requeridos}

\section{Plantilla de la extracción de datos -Los MetaDatos-}

Después de precisar los artículos seleccionados para el estudio, se procede a diseñar las plantillas para estructurar y organizar los datos y así poder extraer la información crítica para el estudio. La extracción de los datos se realiza con base a cuatro plantillas principales:

- Plantilla de extracción de datos de los artículos obtenidos de Bibliotecas Digitales, información general. Los Metadatos de esta plantilla son: <<ID $>>$ $<<$ Título del artículo $>><<$ Nombre de la Revista $><<$ Volumen de revista $>$ $\langle<\langle$ Número de revista $>><$ Primer autor $>\quad<<$ Año de publicación $>>$ $<<$ Nombre de la Biblioteca Digital $>>$ $<<$ Pregunta de investigación asociada $>>$ Plantilla de extracción de datos de los artículos obtenidos de Bases de datos indexadas, información general. Los Metadatos de esta plantilla son: <<ID $>>$ $<<$ Título del artículo $>><<$ Primer autor $>><$ Año de publicación $>>$ $<<$ Nombre de la Base de datos $>>$ $<<$ Pregunta de investigación asociada $>>$

- Plantilla de extracción de datos de los artículos obtenidos, palabras clave. Los Metadatos de esta plantilla son: <<ID $>>$ $<<$ Título del artículo $>><$ Palabras clave de la Biblioteca Digital $>>$ $<<$ Palabras clave indexadas $>$ $<<$ Palabras clave del autor $>><<$ Palabras clave del contenido $>$ 
- Plantilla de extracción de datos de los artículos obtenidos, citas y uso. Los Metadatos de esta plantilla son: $\langle<\mathrm{ID}\rangle\rangle$ $<\langle$ Título del artículo $>>\quad<<$ Citas Crossref $>><<$ Citas Scopus $>><<$ Citas Web of Science $>><<$ Total de Citas $>>$ $<<$ Cantidad de uso en el año 2017>> $<<$ Cantidad de uso en el año 2018>> $<<$ Cantidad de uso en el año 2019>> $<<$ Cantidad de uso en el año 2020>> $<<$ Total de cantidad de uso $>>$

Los datos son almacenados en hojas de cálculo de Microsoft Office Excel permitiendo un análisis de datos fácil y eficaz para la extracción de la información. Es importante mencionar que existen datos en las plantillas que no aplican para todos los artículos dependiendo de la fuente científica de procedencia.

\section{Sintetizar Datos}

Una vez seleccionados los artículos científicos para el estudio, se procede a sintetizar la información recabada, concretando las respuestas a las preguntas de investigación planteadas al principio de la RSL.

A continuación, se presenta la síntesis, a partir de 12 artículos, en relación con la Pregunta 1 (P1) de la RSL ¿Cuál es el papel de la Gestión de Proyectos en la actualidad?

Los proyectos de desarrollo de software a gran escala tienen éxito más a menudo cuando se utilizan métodos ágiles. Se afirma que, el soporte empírico para el uso de métodos ágiles es eficiente tanto en grandes proyectos de software como en los más pequeños, especialmente cuando se incluye un alcance flexible, la entrega frecuente a producción y contextos con alto soporte a los cambios (Jorgensen , 2019).

El éxito de la gestión de proyectos depende de la eficiente de gestión de los mismos. Es esencial monitorear la ejecución de una iteración y prever la capacidad de entregar productos de calidad a medida que avanza la iteración durante la vida del proyecto. Se presenta un enfoque novedoso basado en datos históricos para proporcionar soporte automatizado para gerentes de proyecto y otros tomadores de decisiones en la predicción de la capacidad de entrega para una iteración continua (Choetkiertikul, Dam, Tran, Ghose, \& Grundy, 2018).
El autor, comparte experiencias que ha tenido mientras participaba en el desarrollo de la profesión de gestión y mantenimiento de proyectos. Resalta la importancia de reflexionar sobre experiencias, aprendiendo a aprender y manteniéndose al tanto de lo está sucediendo en el campo de la profesión de la gestión de proyectos. Es la mejor recomendación para el éxito de cualquier practicante (Bierwolf, 2017). Para realizar una gestión de proyectos es necesario mantenerse al día de estos hallazgos recientes del área. Como gerentes de proyecto, necesitamos ser consciente de los últimos métodos, herramientas, tendencias y técnicas para ayudar a la entrega del proyecto o gestión de proyectos $(\mathrm{Ng}$, Understanding Project Management Directions from Project Management Trends, 2019).

El papel del gestor de proyectos (PM) tiene siempre la responsabilidad de entender bien el proyecto general lo suficiente para poder hacer decisiones responsables. La práctica y el conocimiento del PM necesita evolucionar. El PM entiende el impacto tanto de lo previsto como de lo imprevisto y debe ser consciente de posibles consecuencias no deseadas. Hay muchas preocupaciones y preguntas relacionadas a cómo gestionar proyectos (Desmond, What Does the Future Hold for Project Managers?, 2018).

Es difícil aplicar medidas de éxito del proyecto a todos los tipos de proyectos. Discriminar entre el éxito del proyecto y el desempeño del gerente del proyecto es difícil también. En este documento, se presenta una medida genérica de éxito para cualquier proyecto (Meredith \& Zwikael , 2019).

Se expone una herramienta preventiva para aumentar la probabilidad de éxito de los proyectos de alto riesgo llamada Gateway Review Method con un enfoque en los aspectos de aprendizaje en lugar de los aspectos de control. Incluye tres dilemas relacionados con los entornos de planificación y cambio dinámico: La estrategia de cambio utilizada; La estructura de gobierno; y La estructura de gestión de riesgos e incertidumbres (Frijns , Bierwolf, \& van Leeuwen, 2018).

Plantea un enfoque híbrido en el desarrollo de proyectos de software, incluso cuando las compañías tienen establecido un proceso particular. 
Estos enfoques han surgido de la evolución de diferentes prácticas laborales y fueron utilizados con éxito independientemente del tamaño de la compañía o sector industrial (Kuhrmann, y otros, 2019).

Permite explorar los factores de riesgo que amenazan los proyectos de tecnología de la información (TI) de Zimbabwe y desarrolla un modelo sistémico que demuestra cómo están interconectados los factores de riesgo identificados. El modelo sistémico consta de cuatro fases principales: generación de ideas, clarificación de ideas, estructuración de ideas e interpretación de las ideas estructuradas. El resultado del proceso de gestión interactiva es un dígrafo que muestra las relaciones causales entre los riesgos identificados. Se identificaron seis factores de riesgo a través del proceso de gestión interactiva: conocimientos informáticos limitados, mala comunicación, falta de apoyo ejecutivo, complejidad de diseño, burocracia y rotación de empleados (Kanjanda \& Tuan, 2020).

Define un enfoque para la gestión de la incertidumbre y describe estrategias que permiten a los miembros del equipo formalizar y gestionar explícitamente la incertidumbre en proyectos de software (Marinho, Sampaio, \& Moura , 2018).

Presenta un mecanismo que puede proporcionar una mayor comprensión de las interrelaciones causales subyacentes asociadas con el éxito del Proyecto IS y la transición exitosa a las operaciones. Destaca los beneficios de un enfoque interpretativo donde las interrelaciones de los factores IS se pueden modelar para demostrar la influencia potencial en otros factores, por lo tanto, aumentando las posibilidades de éxito del proyecto (Hughes, Rana, \& Dwivedi, 2019).

Explora los impulsores para mantener la consistencia dentro de los procesos de negocios que son altamente compatibles con la configuración del sistema, mientras que la arquitectura del sistema se ve afectada por un cambio técnico en la organización que utiliza métodos tradicionales y ágiles de gestión del cambio (Bogumił, 2020).
Con respecto a la segunda pregunta (P2) ¿Cuáles son los atributos relacionados con la Gestión Ágil de Proyectos? se tienen 10 artículos relacionados. En seguida, se presenta la síntesis de los mismos.

Abrar, et al., realiza una revisión sistemática de la literatura identificando motivadores para adoptar métodos ágiles a gran escala desde una perspectiva de gestión. Los 21 motivadores se extraen de una muestra de 58 trabajos de investigación identificados a través de un proceso SLR. Los factores que se registraron como críticos en todas las variables son: un fuerte apoyo ejecutivo, capacitación y aprendizaje en un entorno de desarrollo ágil, experiencia en desarrollo ágil, competencia de equipo e información de la alta dirección sobre ágil (Abrar, y otros, 2019). En resumen, propone aplicar la mentalidad ágil (Smart, 2018):

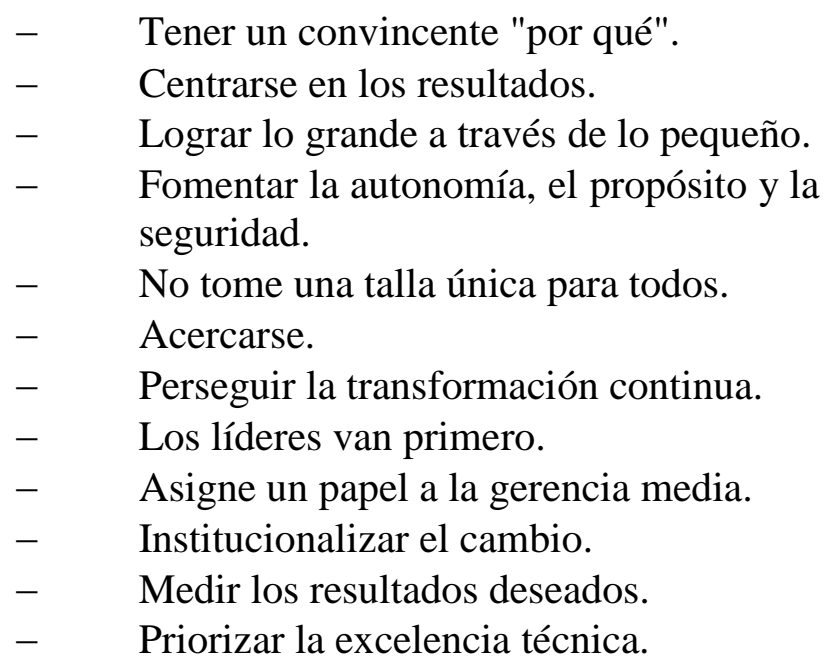

Cada vez más empresas están adoptando métodos ágiles a la medida y mezclando técnicas ágiles en un marco tradicional para la gestión de proyectos. Los equipos de proyecto enfatizan en la flexibilidad para abarcar el contexto del proyecto local (Schmitz, Mahapatra, \& Nerur, 2019).

Proporciona un mapeo sistemático de los marcos, problemas y factores disponibles que afectan una transición ágil exitosa y un proceso de adopción. Se han considerado 28 estudios primarios. Se obtiene una lista de 154 factores situacionales que afectan el proceso ágil de transición y adopción (Jovanović, Mesquida, Mas, \& Colomo-Palacios , 2020). Formula un marco de adaptación de procesos para adaptar los procesos de desarrollo de software basados en la agilidad (Akbar, 2019). 
La caja de herramientas del juego colaborativo contiene un conjunto de juegos para ser utilizados por los miembros del equipo durante una reunión con el objetivo principal de mejorar la comunicación, la cohesión y la coordinación.

Juego 1: Definición de los principios del equipo. Juego 2: Futuras publicaciones de Facebook.

Juego 3: Lecciones aprendidas.

Juego 4: Cronología de picos y valles.

Juego 5: Matriz de expectativas de roles.

Juego 6: Roles que jugamos.

Juego 7: Speed car.

Juego 8: Starfish.

Juego 9: El equipo es, no es, no lo hace.

Juego 10: Comprender el conocimiento del grupo.

Juego 11: Teléfono visual.

Juego12: Pasos de quién-qué-cuándo a la acción.

La caja de herramientas del juego colaborativo que propone el autor se compone de tres componentes principales: un conjunto de tarjetas que describen los 12 juegos presentados anteriormente, el conjunto de activos necesarios y los materiales para ejecutar cada juego y un sitio web para reunir toda la información sobre los juegos y compartir experiencias sobre la adopción por equipos (Mesquida, Jovanović, Jovanović, \& Mas, 2019).

Las principales empresas consultoras internacionales prescriben un desarrollo ágil, particularmente para contextos donde el aprendizaje y la innovación son clave. El autor menciona a Scrum como el marco más común para el desarrollo de software en la mayoría de los países, y se utilizan ampliamente otros métodos como la programación extrema (XP), elementos de desarrollo de software Lean y Kanban. Los métodos de desarrollo ágil han despertado interés principalmente en la ingeniería de software, pero también en otras disciplinas, incluidos los sistemas de información y la gestión de proyectos (Dingsoeyr, Falessi , \& Power, 2019).

Análisis de sistemas y cursos de diseño que utilizan metodologías de desarrollo ágiles. Exhibe una breve descripción pedagógica de la gamificación como un método para atraer a los estudiantes e imprimir conceptos ágiles importantes en la psique del estudiante (Bartholomew, 2017).
Muestra cómo los métodos ágiles se adaptaron y complementaron con prácticas de métodos tradicionales para manejar la escala.

El estudio revelador también sugiere mejoras de una agenda de investigación para el desarrollo ágil a muy gran escala (Dingsøyr, Moe, Fægri , \& Seim , 2018).

Presenta el diseño de un proceso de aprendizaje para promover el trabajo en equipo mediante el aprendizaje basado en proyectos y el concepto de desarrollo de software ágil.

El modelo consta de seis partes: preparación; división del grupo; aprendizaje basado en proyectos; un proceso de trabajo ágil; trabajo en equipo; y un proceso de evaluación derivado del resultado de la evaluación de la idoneidad del estilo de aprendizaje (Sakulviriyakitkul, Sintanakul, \& Srisomphan, 2020).

Ahora se exterioriza la síntesis de los 3 artículos relacionados con la Pregunta 3 (P3) ¿Cómo se adopta el estándar PMBOK en la Gestión Ágil de Proyectos?

Se realiza un análisis comparativo de los estándares aplicados a la gestión de proyectos PMBOK, PRINCE2 e ITIL. Los objetivos de la revisión son: primero, tener una comprensión rápida de los marcos de gestión de proyectos ampliamente utilizados; y en segundo lugar, proporcionar el cómo estos marcos se pueden usar juntos y complementarse entre sí en las diferentes fases del ciclo de vida de un proyecto para abordar las necesidades específicas de la industria con la gestión de productos o servicios de TI (Ng, Tailoring a Project Management Methodology That Suits One's Needs, 2018).

Propone una técnica de retroalimentación instructiva para ser empleada en una unidad de instrucción (UI) que enseña herramientas de gestión de proyectos (PM) alineadas con el cuerpo de conocimiento de gestión de proyectos. Este estudio presenta un experimento para evaluar la contribución de una técnica de retroalimentación instructiva cuando se aplica en la UI para enseñar herramientas de PM alineadas con el estándar PMBOK. Se demuestra que la técnica propuesta puede aumentar percepción y motivación de aprendizaje de los estudiantes (Gonçalves, von Wangenheim, Hauck, \& Zanella, 2018). 
El PMBOK y metodologías de gestión de proyectos ágiles, se presentan de forma comparativa, basadas en un análisis FODA. El primero tiene un enfoque más tradicional, propuesto por el PMI (Project Management Institute) y el segundo se caracteriza por un enfoque más innovador, actual y flexible. Reflexionando con un caso de estudio acerca de las características de la metodología que fueron esenciales para adaptar el método AGILE en el desarrollo del proyecto (Dias de Freitas, Cid de Araujo, \& Braga, 2019).

Por último, se sintetizan los 15 artículos relacionados con la Pregunta 4 (P4) ¿Cómo llevar a cabo una Gestión Ágil de Proyectos en el Desarrollo de Software como parte de la Formación Académica Universitaria?

El estudio exploratorio realizado sugiere que se necesita más investigación sobre el uso in situ del lenguaje Scrum en lugares de trabajo de ingeniería para mejorar e informar a los profesionales de ingeniería sobre las expectativas comunicativas de Scrum. Asimismo, habilitar la mejor ingeniería de educadores de comunicación para preparar futuros ingenieros para aplicar Scrum (Friess, 2019).

Se resume observaciones importantes del plan de riesgos y el registro de los interesados. El plan de riesgos proporciona al equipo excelente oportunidad de profundizar en lo posible la dirección del proyecto, y permite la preparación más efectiva del mismo. El registro de los interesados es un documento que puede ir más allá, identificando lo que el equipo quiere o debe hacer con cada parte interesada, y los planes para lograr esto (Desmond, Project management tools-beyond the basics, 2017).

Descubre que los programas diseñados para un propósito, pero usado para múltiples, no se adapta bien a todas las aplicaciones y, de hecho, puede no ser el mejor ajuste para cualquiera de ellos aparte del que inicialmente fue diseñado. MS Project es probablemente el más programa popular en uso hoy para la gestión de proyectos. Se necesario usar herramientas de uso específico para la gestión de proyectos (Desmond, Project management tools-software tools, 2017).
Demuestra que la gestión ágil de proyectos es exitosa en proyectos pequeños debido a sus características de planificación adaptativa, desarrollo evolutivo y respuesta flexible a los cambios. Estudiantes aprendieron sobre la metodología Scrum y los implementaron cuando trabajaron en su proyecto grupal y con aquellos productos que vinculan con la industria (Ding, Yousef, \& Yue, 2017).

Experiencias de la implementación de técnicas de gestión del aula basadas en Scrum, destinadas principalmente a lograr que los estudiantes asuman más responsabilidad por su aprendizaje. Presenta las lecciones aprendidas durante la implementación de nuevas ideas en la gestión del curso usando la gestión ágil (Duvall, Hutchings, \& Kleckner, 2017).

Asesores de la industria indicaron la necesidad de que los estudiantes de ciencias de la computación de la Universidad Western New England tengan una mayor exposición a herramientas de desarrollo de software, diseño de software y patrones arquitectónicos para estar mejor calificados para pasantías e ingresar a la fuerza laboral. Estas recomendaciones hicieron eco de las pautas del plan de estudios de ACM que sugieren la exposición a las mejores prácticas de desarrollo de software. Motiva a la revisión de planes de estudio (O'Neill, 2018).

Encuentra que los recién graduados universitarios que ingresan a la industria del desarrollo de software encuentran dificultades al principio de sus carreras, debido a diferencias significativas entre sus experiencias de codificación en la academia y lo que se espera de ellos en el trabajo. Explora la brecha entre el desarrollo de software académico y de la industria (Craig, Conrad, Lynch, Lee, \& Anthony, 2018).

Analiza: La importancia de las actitudes de los estudiantes y cómo influyen en su elección de especializaciones en la universidad; cómo usar la Escala de Actitud hacia la Tecnología de la Información; diferentes estrategias que se han implementado para tener un impacto positivo en las actitudes de los estudiantes hacia la informática; y cómo adaptar las estrategias probadas para su uso en diferentes instituciones y realizar más investigaciones (Gokhale, 2018). 
El Aprendizaje Basado en Proyectos para proporcionar una experiencia acumulativa diseñada para incluir un enfoque claro en: pensamiento crítico y resolución de problemas; colaboración y liderazgo; comunicaciones verbales y escritas; y trabajo independiente. discute la aplicación del aprendizaje basado en proyectos a la educación en Ciencias de la Computación, proporcionando la perspectiva de un estudiante y la perspectiva de un asesor de investigación (McManus \& Costello, 2019).

Tanto los estudiantes como el profesorado ofrecen lecciones aprendidas y nuestras sugerencias de los "10 principales" para mantener un gran esfuerzo de software en múltiples grupos de estudiantes (Feng \& LeBlanc, 2019).

Presenta una propuesta para la lista de temas que debe cubrir el MBE (Model-Based Software Engineering)-BOK (Body of Knowledge). Como tal, tiene como objetivo caracterizar los contenidos y las prácticas conocidas de la disciplina Model-Based Software Engineering (MBE), ayudar a las universidades y otras instituciones que imparten cursos de enseñanza sobre SE para desarrollar sus planes de estudio MBE e identificar el conjunto básico de conceptos que cualquier ingeniero MBE debe conocer, proporcionando una terminología de referencia común y consistente (Burgueño, y otros, 2019).

Presenta metodologías, herramientas y técnicas de gestión de proyectos más utilizadas por los gerentes de proyectos, que se pueden aplicar en nuestro día a día, como la Guía del cuerpo de conocimiento de gestión de proyectos (PMBOK), Estructura de desglose del trabajo (WBS), MS Project, Project Canvas y la metodología ágil con el marco Scrum (Hirata \& Bortoletto, 2019).

Los equipos están aumentando su uso de la metodología de proceso Kanban en una variedad de proyectos de sistemas de información, incluyendo desarrollo de software y proyectos de ciencia de datos. Informa sobre la exploración del uso de un entrenador Kanban, con respecto a cómo el entrenador podría interactuar con el equipo y cómo el uso de un entrenador impacta los resultados del equipo (Shamshurin \& Saltz, 2019).
Identifica cómo se ha hecho la asociación de las metodologías de desarrollo de software ágil con los modelos de madurez comúnmente utilizados en la metodología tradicional. Documenta qué modelos de madurez se utilizan y cómo se relacionan con la práctica del desarrollo ágil de software (Araújo Camargo, Gal, Macorin de Azevedo, \& Souza das Neves, 2020).

Se enseñan y utilizan muchos enfoques diferentes de desarrollo de productos en las disciplinas de ingeniería y gestión. Estos métodos, procesos y entornos de diseño formalizados difieren en los tipos de proyectos para los que son relevantes, los componentes del proyecto que incluyen y el soporte que brindan a los usuarios. Este artículo detalla una revisión de dieciséis enfoques de desarrollo de productos bien establecidos, el desarrollo de un sistema de soporte de decisiones para ayudar a los diseñadores y gerentes a navegar estos enfoques. Los enfoques incluidos: pensamiento de diseño, pensamiento de sistemas, gestión de calidad total, desarrollo ágil, proceso en cascada, diseño de ingeniería, modelo en espiral, modelo en $\mathrm{V}$, diseño axiomático, diseño basado en valores, diseño basado en decisiones, fabricación ajustada, Six Sigma, teoría de restricciones, scrum y programación extrema: se clasifican en función de seis criterios: complejidad, orientación, fase, aplicabilidad de hardware o software, valores y usuarios (Stewart, Giambalvo, \& Vance, 2020).

\section{Fase III: Documentar la RSL}

\section{Informe de Revisión}

Los hallazgos del análisis se presentan en el apartado de Resultados.

\section{Validación del Informe}

La validez descriptiva es la medida en que las observaciones se describen de manera precisa y objetiva. En este sentido se utilizan formularios de extracción diseñados en VBA (Visual Basic para Aplicaciones) en Excel para el registro de los datos en las plantillas de extracción descritas en el apartado correspondiente y así mitigar el sesgo de la información obtenida. 
Finalmente, con respecto a la validez interpretativa, que refiere a la conexión de conclusiones con los datos se realiza un análisis de lectura profunda con el conjunto de artículos científicos seleccionados por pregunta de investigación, dejando de lado posibles datos no justificados en el presente análisis.

\section{Resultados}

Después de realizar el análisis de los artículos científicos recopilados se da respuesta a las preguntas de investigación definidas. Esto constituye el Informe de Revisión en la metodología aplicada. Enseguida se exponen las respuestas por cada pregunta de investigación del presente estudio.

P1: ¿Cuál es el papel de la Gestión de Proyectos en la actualidad?

Los autores de los artículos asociados a esta pregunta enfatizan en la importancia de aplicar metodologías ágiles o híbridas en la gestión de proyectos para alcanzar el éxito de los mismos. Asimismo, el gestor de proyectos debe mantener el interés del avance y evolución de la gestión de proyectos y aprender de experiencias e iteraciones históricas.

En conjunto, se reconocen diversos factores que afectan directamente el logro del éxito del proyecto, entre los cuáles se pueden mencionar, falta de apoyo ejecutivo o de gobierno, burocracia, mala comunicación y la falta de gestión de riesgos e incertidumbres.

P2: ¿Cuáles son los atributos relacionados con la Gestión Ágil de Proyectos?

Primeramente, se considera los métodos ágiles a la medida y mezclando técnicas ágiles en un marco tradicional para la gestión de proyectos. Lo anterior, acuña atributos específicamente alineados en su mayoría a los principios del manifiesto ágil, entre los cuales, los autores insisten en destacar: conocer el "por qué" (metas), centrarse en los resultados, fomentar la autonomía, trabajo colaborativo, medir los resultados deseados, fuerte apoyo ejecutivo, capacitación y aprendizaje del entorno de desarrollo ágil, experiencia en desarrollo ágil, competencia de equipo, informar a la alta dirección sobre ágil, estandarizar la documentación del proyecto, reducir la documentación, recibir comentarios o retroalimentación activamente.

\begin{abstract}
Aumentar el número de micro iteraciones, formalizar el patrón de comunicación del proyecto, fomentar la comunicación abierta del proyecto y seleccionar herramientas apropiadas.
\end{abstract}

P3: ¿Cómo se adopta el estándar PMBOK en la Gestión Ágil de Proyectos?

Se presenta específicamente una técnica de retroalimentación instructiva para la enseñanza de una Unidad Instruccional alineada con el estándar PMBOK y el uso de aplicado de herramientas. De igual forma, se analizan los estándares PMBOK, PRINCE2 e ITIL, comparando sus beneficios y el uso combinado de los mismos para una gestión de proyectos más eficiente y eficaz.

También se estudia el PMBOK y metodologías de gestión de proyectos ágiles, de forma comparativa, basadas en un análisis FODA (Fortalezas, Oportunidades, Debilidades, Amenazas) donde, sin lugar a dudas, este estándar es recomendado para la gestión ágil de proyectos.

P4: ¿Cómo llevar a cabo una Gestión Ágil de Proyectos en el Desarrollo de Software como parte de la Formación Académica Universitaria?

Los autores coinciden en que se enseñan y utilizan muchos enfoques diferentes de desarrollo de productos en las disciplinas de ingeniería y gestión. Se encuentran experiencias de la implementación de técnicas de gestión del aula basadas en Scrum, destinadas principalmente a lograr que los estudiantes asuman más responsabilidad por su aprendizaje logrando una gestión ágil de proyectos exitosa en proyectos pequeños. A su vez, se analiza una propuesta para la lista de temas que debe cubrir el MBE (Model-Based Software Engineering)BOK (Body of Knowledge) en la formación académica universitaria. Como tal, tiene el objetivo de caracterizar contenidos y prácticas en cursos de enseñanza. 
Asesores de la industria indicaron la necesidad de que los estudiantes de ciencias de la computación de la Universidad Western New England tengan una mayor exposición a herramientas de desarrollo de software, diseño de software y patrones arquitectónicos para estar mejor calificados para pasantías e ingresar a la fuerza laboral con fundamento en el plan de estudios de ACM (Association for Computing Machinery) que sugieren la exposición a las mejores prácticas de desarrollo de software. En este aspecto, otro estudio encuentra que los recién graduados universitarios que ingresan a la industria del desarrollo de software encuentran dificultades al principio de sus carreras, debido a diferencias significativas entre sus experiencias de codificación en la academia y lo que se espera de ellos en el trabajo, es decir, existe una brecha entre el desarrollo de software académico y de la industria.

Entre las metodologías, herramientas y técnicas de gestión de proyectos más utilizadas por los gerentes de proyectos se encuentran la Guía del cuerpo de conocimiento de gestión de proyectos (PMBOK), Estructura de desglose del trabajo (WBS), MS Project, Project Canvas y la metodología ágil con el marco Scrum, resaltando en este último enfatizar el uso in situ del lenguaje Scrum y mejorar la habilidad de los educadores para preparar futuros ingenieros para aplicar esta metodología.

Por último, no siendo de menor importancia, fortalecer las actitudes de los estudiantes y analizar cómo llevan a cabo su elección de especializaciones en la universidad dirigiendo su interés hacia la Tecnologías de la Información usando diferentes estrategias para tener un impacto positivo en las actitudes de los estudiantes hacia la informática.

\section{Agradecimiento}

Agradecemos al Mtro. José Román Ruiz Carrillo, Rector de la Universidad Autónoma de Campeche por la disposición y apoyo brindado para la publicación del presente artículo. De igual forma, se extiende el reconocimiento al Mtro. Guadalupe Manuel Estrada Segovia, Director de la Facultad de Ingeniería y la Mtra. Nancy Georgina Ortiz Cuevas, Coordinadora del Programa Educativo Ingeniería en Sistemas Computacionales por la empatía por impulsar los estudios de investigación en el área en Ciencias de la Computación.

\section{Conclusiones}

La metodología de la revisión sistemática de la literatura es completamente aplicable a investigaciones en el campo profesionalizante de las ciencias de la computación contribuyendo particularmente, en gran medida, al conocimiento en la formación disciplinar, atendiendo al avance dinámico y creciente que la innovación y la tecnología sufren en el mundo actual. Como resultado de la presente revisión sistemática de la literatura se puede afirmar que la gestión ágil de proyectos es una competencia profesionalizante indispensable para la formación académica universitaria que involucre habilidades de la ingeniería de software. Al igual de requerir la definición y uso de estándares reconocidos $\mathrm{y}$ aceptados internacionalmente como el PMBOK y el uso de herramientas específicas para su ejecución.

\section{Referencias}

Abrar, M. F., Khan, M. S., Ali, S., Ali, U., Majeed, M. F., Ali, A., . . Rasheed, N. (2019). Motivators for Large-Scale Agile Adoption From Management Perspective: A Systematic Literature Review. IEEE Access, 7, 2266022674.

Akbar, R. (2019). Tailoring Agile-Based Software Development Processes. IEEE Access, 7, 139852-139869,.

Araújo Camargo, K. G., Gal, N. V., Macorin de Azevedo, M., \& Souza das Neves, J. M. (2020). Study on the association of maturity models and agile methodologies. Research, Society and Development., 9(2), e115922169-e115922169. doi:10.33448/rsd-v9i2.2169

Bartholomew, K. W. (2017). Agile gamification: activities and techniques to create a winning learning environment. Journal of Computing Sciences in Colleges, 33(2), 57-58.

Bierwolf, R. (2017). Towards project management 2030: Why is change needed? IEEE Engineering Management Review, 45(3), 21-26.

Bogumił, H. (2020). Dissimilarities between applied methods of project management impacting regression in business processes and technical architecture. Journal of Entrepreneurship, Management and Innovation, 16(1), 133-168. doi:10.7341/20201615

HERNÁNDEZ-CRUZ, Luz María, CASTILLO-TÉLLEZ, Margarita, MEX-ALVAREZ, Diana Concepción y CAB-CHAN José Ramón. La gestión ágil de proyectos de software en la formación Académica Universitaria: Una revisión sistemática de la literatura. Revista de Tecnología y Educación. 2020 
Burgueño, L., Ciccozzi, F., Famelis, M., Kappel, G., Lambers, L., Mosser, S., . . . Wimmer, M. (2019). Contents for a Model-Based Software Engineering Body of Knowledge. Software and Systems Modeling, 18, 3193-3205. doi:https://doi.org/10.1007/s10270-019-007469

Choetkiertikul, M., Dam, H. K., Tran, T., Ghose, A., \& Grundy, J. (2018). Predicting Delivery Capability in Iterative Software Development. IEEE Transactions on Software Engineering, 44(6), 551-573.

Craig, M., Conrad, P., Lynch, D., Lee, N., \& Anthony, L. (2018). Listening to early career software developers. Journal of Computing Sciences in Colleges, 33(4), 138-149.

Desmond, C. (2017). Project management toolsbeyond the basics. IEEE Engineering Management Review, 45(3), 25-26.

Desmond, C. (2017). Project management toolssoftware tools. IEEE Engineering Management Review, 45(4), 24-25.

Desmond, C. (2018). What Does the Future Hold for Project Managers? IEEE Engineering Management Review, 46(4), 14-15.

Dias de Freitas, M., Cid de Araujo, F. C., \& Braga, S. L. (2019). Comparative analysis of project management methodologies PMBOK and agile - A case study with companies of the Brazilian energetic sector. evista GEINTEC., 9(3), 4993-5007. doi:10.7198/geintec.v9i3.1340

Ding, D., Yousef, M., \& Yue, X. (2017). A case study for teaching students agile and scrum in Capstone course. Journal of Computing Sciences in Colleges, 32(5), 95-101.

Dingsoeyr, T., Falessi , D., \& Power, K. (2019). Agile Development at Scale: The Next Frontier. IEEE Software, 36(2), 30-38.

Dingsøyr, T., Moe, N. B., Fægri , T. E., \& Seim , E. A. (2018). Exploring software development at the very large-scale: a revelatory case study and research agenda for agile method adaptation. Empirical Software Engineering, 23, 490-520. doi:https://doi.org/10.1007/s10664-017-9524-2
Duvall, S., Hutchings, D., \& Kleckner, M. (2017). Changing perceptions of discrete mathematics through scrum-ased course management practices. Journal of Computing Sciences in Colleges, 33(2), 182-189.

Feng, W., \& LeBlanc, M. D. (2019). Top-10 suggestions from a decade of managing undergraduate software teams. Journal of Computing Sciences in Colleges, 34(6), 70-83.

Friess, E. (2019). Scrum Language Use in a Software Engineering Firm: An Exploratory Study. IEEE Transactions on Professional Communication, 62(2), 130-147.

Frijns , P., Bierwolf, R., \& van Leeuwen, F. (2018). Dilemmas in managing projects in a dynamic environment. IEEE Engineering Management Review, 46(1), 19-23.

Gokhale, A. (2018). Measuring students' attitudes toward information technology: conference tutorial. Journal of Computing Sciences in Colleges, 33(4), 101-102.

Gonçalves, R. Q., von Wangenheim, C. A., Hauck, J. C., \& Zanella, A. (2018). An Instructional Feedback Technique for Teaching Project Management Tools Aligned With PMBOK. IEEE Transactions on Education, 61(2), 143 - 150. doi:10.1109/TE.2017.2774766

Hirata, E., \& Bortoletto, W. W. (2019). Planning our everyday using tools and techniques of project management. Iberoamerican Journal of Project Management., 10(2), 14-29.

Hughes, D., Rana, N. P., \& Dwivedi, Y. K. (2019). Elucidation of IS project success factors: an interpretive structural modelling approach. Annals of Operations Research, 285, 35-66.

Jorgensen, M. (2019). Relationships Between Project Size, Agile Practices, and Successful Software Development: Results and Analysis. IEEE Software, 36, 39-43.

Jovanović, M., Mesquida, A.-L., Mas, A., \& Colomo-Palacios , R. (2020). Agile Transition and Adoption Frameworks, Issues and Factors: A Systematic Mapping. IEEE Access, 8, 1571115735 . 
Kanjanda, T., \& Tuan, N.-T. (2020). A Systemic Exploration of the Risk Factors in Zimbabwean Information Technology Projects. Systemic Practice and Action Research, 77-93. doi:https://doi.org/10.1007/s11213-019-095157

Kitchenham, B. A., Budgen, D., \& Brereton, P. (2015). Evidence-Based Software Engineering and Systematic Reviews. New York: CRC Press. Taylor \& Francis Group.

Kuhrmann, M., Diebold, P., Munch, J., Tell, P., Trektere, K., McCaffery, F., . . Prause, C. R. (2019). Hybrid Software Development Approaches in Practice: A European Perspective. IEEE Software, 36(4), 20-31.

Marinho, M., Sampaio, S., \& Moura, H. (2018). Managing uncertainty in software projects. Innovations in Systems and Software Engineering, $\quad 14, \quad 157-181$. doi:https://doi.org/10.1007/s11334-017-0297-y

McManus, J. W., \& Costello, P. J. (2019). Project based learning in computer science: a student and research advisor's perspective. Journal of Computing Sciences in Colleges, 34(3), 38-46.

Meredith, J., \& Zwikael , O. (2019). When is a Project Successful? IEEE Engineering Management Review, 47(3), 127-134.

Mesquida, A.-L., Jovanović, J., Jovanović, M., \& Mas, A. (2019). Agile software process improvement: a collaborative game toolbox. IET Software, 13(2), 106-111.

Ng, J. J. (2018). Tailoring a Project Management Methodology That Suits One's Needs. IEEE Engineering Management Review, 46(2), 49-54.

Ng, J. J. (2019). Understanding Project Management Directions from Project Management Trends. IEEE Engineering Management Review, 47(2), 128-132.

O'Neill, B. (2018). Curriculum changes to improve software development skills in undergraduates. Journal of Computing Sciences in Colleges, 33(6), 86-96.
Sakulviriyakitkul, P., Sintanakul, K., \& Srisomphan, J. (2020). The Design of a Learning Process for Promoting Teamwork using ProjectBased Learning and the Concept of Agile Software Development. International Journal of Emerging Technologies in Learning (iJET), 15(03), 207-222. doi:10.3991/ijet.v15i03.10480

Schmitz, K., Mahapatra, R., \& Nerur, S. (2019). User Engagement in the Era of Hybrid Agile Methodology. IEEE Software, 36(4), 32-40.

Shamshurin, I., \& Saltz, J. S. (2019). Using a coach to improve team performance when the team uses a Kanban process methodology. International Journal of Information Systems and Project Management., 7(2), 61-77. doi:10.12821/ijispm070204

Smart, J. (2018). To Transform to Have Agility, Dont Do a Capital A, Capital T Agile Transformation. IEEE Software, 35(6), 56 - 60.

Stewart, S., Giambalvo, J., \& Vance, J. (2020). A Product Development Approach Advisor for Navigating Common Design Methods, Processes, and Environments. Designs, 4(1). doi: $10.3390 /$ designs 4010004 